\title{
Padrões de crescimento econômico dos municípios do MATOPIBA
}

\author{
Economic growth patterns of the MATOPIBA'S municipalities \\ Luiz Carlos de Santana Ribeiro ${ }^{1}$ (1), Ariana Souza Lôbo ${ }^{2}$ (]), Lizandra Duarte da Silva ${ }^{3}$, \\ Nathália Francelina Santos Andrade ${ }^{4}$ (D) \\ ${ }^{1}$ Programa Acadêmico de Pós-graduação em Economia, Universidade Federal de Sergipe (UFS), São Cristóvão (SE), Brasil. \\ E-mail: ribeiro.luiz84@gmail.com \\ ${ }^{2}$ Ministério da Agricultura, Pecuária e Abastecimento, Brasília (DF), Brasil. E-mail: ariana.lobo@agricultura.gov.br \\ ${ }^{3}$ Programa de Pós-graduação em Economia, Universidade Federal de Juiz de Fora (UFJF), Juiz de Fora (MG), Brasil, \\ E-mail: lizandra-duarte@hotmail.com \\ 4Universidade Federal de Sergipe (UFS), São Cristóvão (SE), Brasil. E-mail: nathaliaseguro@gmail.com
}

Como citar: Ribeiro, L. C. S., Lôbo, A. S., Silva, L. D., \& Andrade, N. F. S. (2020). Padrões de crescimento econômico dos municípios do MATOPIBA. Revista de Economia e Sociologia Rural, 58(3), e212613. https://doi.org/10.1590/18069479.2020 .212613

Resumo: Este artigo objetiva classificar os municípios do MATOPIBA com base em um conjunto de variáveis socioeconômicas, com destaque para as características agrícolas. Além disso, propõe-se a decompor o crescimento do VAB setorial no período 2010-2015, no sentido de identificar grupos de municípios com vantagens competitivas e de especialização. Para tanto, foram utilizadas conjuntamente a análise de cluster e o shift-share com base em informações do IBGE, RAIS e PNUD. A aplicação do shift-share foi realizada com base nos grupos formados pela análise de cluster, o que permite maior integração dos métodos. Os principais resultados mostram a formação de cinco grupos, sendo apenas um deles considerado incipiente do ponto de vista do dinamismo da agropecuária. Os resultados do shift-share apontam que esse grupo considerado incipiente foi o único a apresentar VLT negativa e não apresentar vantagem competitiva nem ser especializado na agropecuária.

Palavras-chave: MATOPIBA, agropecuária, cluster, shift-share.

\begin{abstract}
This paper aims to classify the municipalities of MATOPIBA based on a set of socioeconomic variables, mainly agricultural characteristics, as well as to decompose the growth of the sectorial GVA in the period 2010-2015, in order to identify groups of with competitive advantages and specialization. It was also used cluster and shift-share analysis based on information from IBGE, RAIS and UNDP. More specifically, the shift-share will be realized based on the groups formed by the cluster analysis, which allows a better integration of the methods. The main results show the formation of five groups, being only one of them considered incipient from the point of view of the agriculture dynamism. Shift-share results indicate that this group considered incipient was the only one to present negative VLT and did not present competitive advantage nor to be specialized in Agriculture.
\end{abstract}

Keywords: MATOPIBA, agriculture, cluster, shift-share.

\section{Introdução}

Durante os anos de 1970, o cerrado brasileiro foi bastante explorado pela produção agrícola e pecuária. A primeira grande expansão ocorreu no estado do Mato Grosso, considerado a fronteira agrícola da época. Na medida em que as cidades foram se estruturando e as culturas se tornaram mais fortes, outras regiões começaram a ser exploradas, principalmente a antiga vila conhecida como Mimoso do Oeste na Bahia, em torno da cidade de Barreiras. Com o tempo, os estados de Maranhão, Tocantins, Piauí e Bahia tornaram-se o centro do agronegócio e passaram a ser conhecidos como MATOPIBA (Horvat et al., 2015). 
A expressão MATOPIBA refere-se a um acrônimo formado com as siglas dos estados do Maranhão, Tocantins, Piauí e Bahia. Essa expressão remete a uma delimitação geográfica que contempla a totalidade do estado de Tocantins e parcialmente as outras três Unidades da Federação. Essa delimitação caracteriza-se pela substancial expansão de uma fronteira agrícola dotada de tecnologias de alta produtividade (Miranda et al., 2014). O MATOPIBA foi oficializado como região por meio do decreto $n^{\circ} 8.447$ de 6 de maio de 2015, que trata do Plano de Desenvolvimento Agropecuário para o MATOPIBA, cuja finalidade era promover e coordenar políticas públicas voltadas ao desenvolvimento econômico sustentável fundado nas atividades agrícolas e pecuárias.

Nos últimos 20 anos, segundo Sá et al. (2015), observou-se grande aumento na produção de soja resultante do crescimento da área plantada e da produtividade. A consequência direta desse processo foi o aumento do PIB dos municípios que constituem a região. No entanto, segundo essas autoras, há concentração da riqueza gerada no MATOPIBA, uma vez que grande parcela da população das cidades localizadas nessa região está à margem dos benefícios gerados pelo agronegócio. A Figura 1 mostra a delimitação territorial do MATOPIBA.
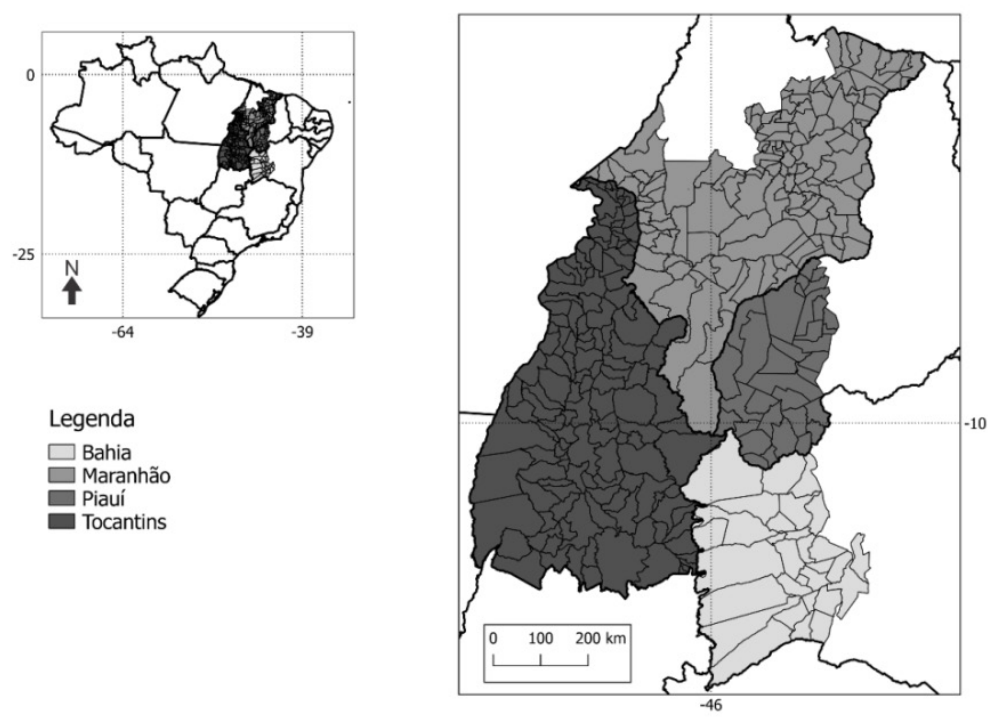

Figura 1: Região do MATOPIBA. Fonte: Elaboração própria a partir da Portaria $n^{\circ}$ 244, de 12 de novembro de 2015 (Brasil, 2015).

De acordo com a Empresa Brasileira de Pesquisa Agropecuária, o MATOPIBA é formado por 31 microrregiões, 337 municípios e ocupa uma área de aproximadamente 73 milhões de hectares.

Ainda que o estado de Tocantins apresente o maior número de municípios do MATOPIBA, o estado do Maranhão, em 2015, era o que apresentava a maior participação no PIB total da região, com $44,5 \%$, seguido por Tocantins com $30,8 \%$. Piauí, por outro lado, é o menor em termos de representatividade do PIB regional, contribuindo com apenas $3,4 \%$, e a Bahia, que apresenta a menor quantidade de municípios, responde por $21,3 \%$ do PIB regional. Em termos de contribuição setorial, o setor de serviços representa $38,9 \%$ do PIB do MATOPIBA, seguido da agropecuária com 20,1\%, da administração pública com $26 \%$ e da indústria com 15\% (Instituto Brasileiro de Geografia e Estatística, 2017). Entre 2010 e 2015, o MATOPIBA apresentou uma taxa de crescimento do PIB real de 5,1\% a.a., bem superior à taxa de crescimento brasileira de $1,5 \%$ a.a.

Sob uma perspectiva furtadiana, Sá et al. (2015) argumentam que ainda que o MATOPIBA venha experimentando uma onda de crescimento econômico, desencadeada principalmente pelo aumento da produtividade dos fatores associados ao agronegócio, isso não tem sido revertido em desenvolvimento. Em outras palavras, para essas autoras, o agronegócio 
praticado no MATOPIBA gera crescimento, mas não desenvolvimento ${ }^{1}$. Uma das razões para dar suporte a essa argumentação é que o uso da mão de obra local é limitado, o que exclui grande parcela da população residente. Mais que isso, Rezende (2006) argumenta que o modelo agrícola moderno prioriza o uso de insumos químicos e bens de capital (recursos escassos) na agropecuária em detrimento da mão de obra. Todavia, o recurso abundante e barato no Brasil é justamente a mão de obra (pouco qualificada), que acaba marginalizada.

Analisando a região sob uma perspectiva social, de acordo com Porcionato et al. (2018), alguns indicadores como o Índice de Desenvolvimento Humano Municipal (IDH-M) e o Índice de Vulnerabilidade Social (IVS) apresentaram evidentes tendências de melhoria social em todos os estados que compõem a região do MATOPIBA. A partir da observação dessas variáveis selecionadas, os autores acreditam que a melhoria do IDH-M está relacionada diretamente à melhoria dos indicadores educacionais, como diminuição da taxa de analfabetismo, aumento da média de anos de estudo da região e aumento da taxa de frequência, principalmente nos anos do ensino médio. Já a melhoria do IVS, por sua vez, associa-se às melhorias na infraestrutura urbana, como o aumento de redes de abastecimento de água, de serviços de esgotamento sanitário e da coleta de lixo no território.

A Embrapa identificou que das 250 mil fazendas localizadas no MATOPIBA registradas no Censo Agropecuário de 2006 do IBGE, 94\% dessas unidades têm como proprietários pessoas pobres (14\%) ou muito pobres (80\%), conforme mostra a Tabela 1.

Tabela 1: Distribuição da renda bruta (rb) em classes de salário mínimo mensal (slmm), frequência, em número de estabelecimentos e respectivas porcentagens, participação de cada classe na renda bruta (\%) e renda bruta mensal por estabelecimento (rb/est./slmm)

\begin{tabular}{ccccc} 
Classes, sm & Frequência & $\%$ & $\%$ rb & rb/est./slmm \\
$(0,2]$ & 199.801 & 79,84 & 5,22 & 0,48 \\
$(2,10]$ & 34.917 & 13,95 & 8,35 & 4,36 \\
$(10,200]$ & 14.500 & 5,79 & 26,74 & 33,64 \\
$>200$ & 1.020 & 0,42 & 59,68 & $1.067,21$ \\
Total & 250.238 & 100 & 100 & 7,29 \\
\hline
\end{tabular}

Fonte: Alves et al. (2015).

A classe muito pobre recebe até 2 salários mínimos mensais, ao passo que a classe pobre recebe entre 2 e 10 salários mínimos. Por outro lado, a classe média recebe entre 10 e 200 salários mínimos e a mais rica ganha acima de 200 salários mínimos. Percebe-se por meio da Tabela 1 que apenas 0,42\% dos estabelecimentos concentram cerca de $60 \%$ da renda bruta total da região do MATOPIBA (Alves et al., 2015).

Para reforçar esse argumento, a Figura 2 apresenta o Coeficiente de Williamson (1965) ${ }^{2}$ calculado a partir do Valor Adicionado Bruto (VAB) total e do VAB da agropecuária dos municípios do MATOPIBA entre 2000 e 2015.

\footnotetext{
${ }^{1}$ Para saber mais sobre a discussão que envolve os conceitos de crescimento e desenvolvimento econômico, ver Bresser-Pereira (2004).

${ }^{2}$ Formalmente, o coeficiente de Williamson pode ser expresso como $V w=\frac{\sqrt{\sum_{i}\left(y_{i}-\bar{y}\right)^{2} \cdot \frac{f_{i}}{n}}}{\bar{y}}$. Em que: $y_{i}$ é a renda per capita do i-ésimo município; y é a renda per capita regional; $f_{i}$ é a população do i-ésimo município; e n é a população regional.
} 


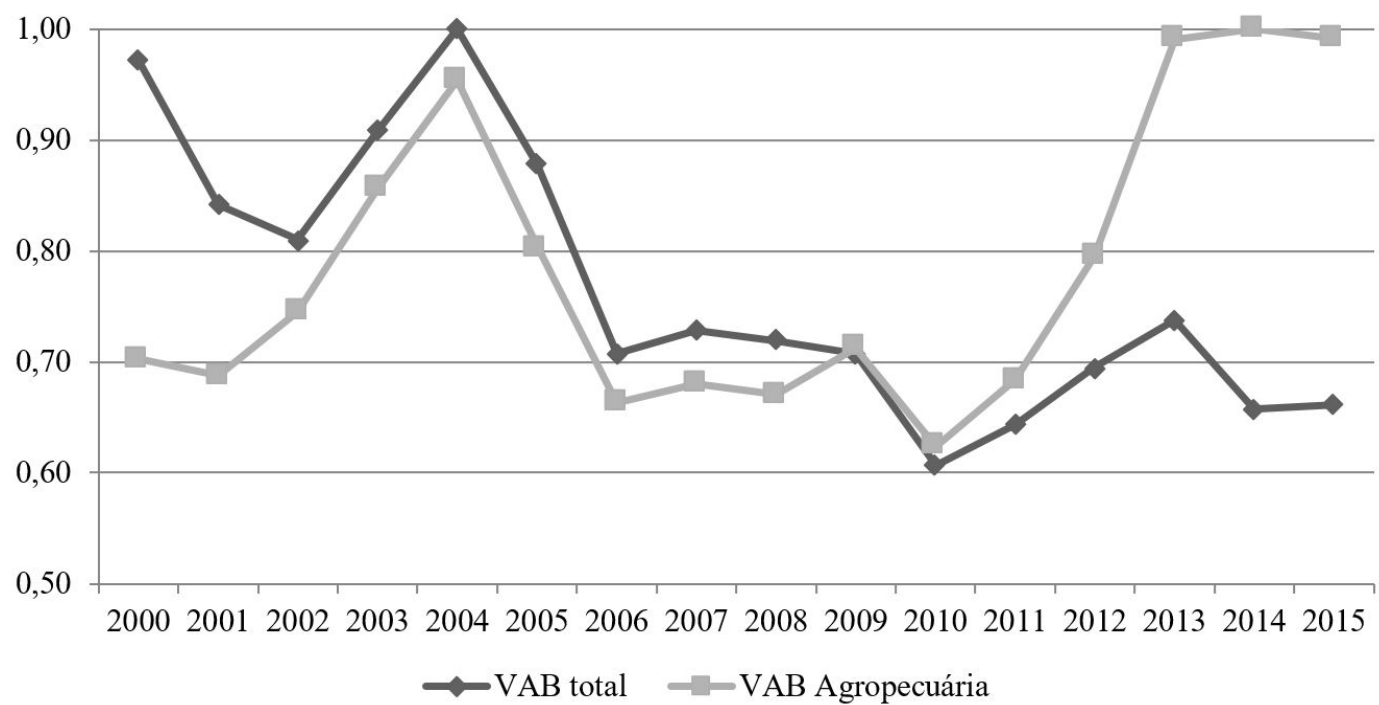

Figura 2: Coeficiente de Williamson normalizado referente ao VAB total e ao VAB da agropecuária do MATOPIBA para o período 2000-2015. Fonte: Elaboração própria a partir de dados do IBGE, 2000-2015.

Quando se considera o VAB total do MATOPIBA, nota-se que a região segue uma trajetória de queda das desigualdades regionais, em consonância com a experimentada pelo Brasil como já apontada por Silveira Neto \& Azzoni $(2011,2012)$ e Ribeiro et al. (2018). No entanto, quando se considera o VAB da agropecuária, o indicador caminha na contramão nacional, ou seja, percebe-se um agravamento das desigualdades intrarregionais. Essa característica, por sua vez, é a principal motivação deste trabalho para investigar os municípios do MATOPIBA.

Dessa forma, observa-se que embora os 337 municípios perteçam a uma mesma região, isso não garante que haja uma similitude socioeconomica entre eles, pelo contrário, percebe-se uma marcante heterogeneidade. Diante disso, tomando por base os estudos de Ribeiro \& Andrade (2015) e Ribeiro \& Lopes (2015), pretende-se utilizar de forma complementar a análise de cluster e o shift-share com o objetivo de classificar os municípios do MATOPIBA com base em um conjunto de variáveis socioeconômicas, em especial características agrícolas. Além disso, decompõe-se o crescimento do VAB setorial no período 2010-2015, no sentido de identificar grupos de municípios com vantagens competitivas e de especialização.

O primeiro método define grupos de municípios da região com base em variáveis socioeconômicas, ao passo que o shift-share irá decompor o crescimento do VAB setorial dos grupos criados pela análise de cluster em componentes que identificarão grupos com vantagens competitivas e com características de especialização em determinado setor de atividade. Nesse sentido, a principal contribuição deste artigo é caracterizar os municípios do MATOPIBA a partir da integração de dois métodos já consolidados na literatura. Espera-se a partir disso contribuir com a formulação de políticas públicas para a região.

\section{Métodos e base de dados}

\subsection{Análise de cluster}

A análise de cluster, também conhecida como análise de agrupamentos ou análise de conglomerados, é uma técnica estatística multivariada em que as variáveis estudadas dependem umas das outras, ou seja, uma técnica de interdependência. A análise de agrupamentos, assim como o próprio nome indica, busca agrupar de maneira homogênea os objetos tendo como critério suas similaridades e características (Mardia et al., 1997).

Segundo Fávero et al. (2009), a análise de cluster busca estudar a estrutura natural dos grupos e pode muitas vezes ser confundida com a análise fatorial, pois as duas técnicas têm como objetivo identificar grupos de variáveis selecionadas. Porém, na análise de cluster a variável estatística de agrupamento é o próprio conjunto de elementos selecionados e que 
não são estimados empiricamente. Esse tipo de técnica identifica e segrega os elementos de forma a reunir em um mesmo grupo objetos semelhantes e homogêneos.

A análise de conglomerados pode ser dividida em algumas etapas, a saber: i) seleção e padronização das variáveis e elementos a serem agrupados; ii) seleção da medida de distância ou semelhança entre os elementos, em que se analisa o quanto os elementos são parecidos ou diferentes entre si; iii) seleção do tipo de método de agrupamento: hierárquico ou não hierárquico; iv) escolha da quantidade de agrupamentos que vão ser formados; e v) interpretação e validação dos agrupamentos formados (Everitt et al., 2001).

Na seleção das variáveis e elementos a serem agrupados, os grupos que serão formados refletirão a estrutura inerente das variáveis selecionadas e podem ser bastante sensíveis quanto à inclusão de variáveis com comportamentos incomuns, ou seja, com a presença de outliers (Fávero et al., 2009).

Uma decisão importante nesse tipo de análise, portanto, implica na escolha das variáveis que possam representar a região ou tema a ser estudado. Desse modo, a seleção de variáveis irrelevantes ou que não se relacionem com o escopo do estudo não ajudaria na classificação dos grupos. Portanto, as variáveis utilizadas neste artigo incluirão aspectos socioeconômicos, majoritariamente relacionados à atividade agrícola, que caracterizam o MATOPIBA (ver seção 3.3).

Como o objetivo da análise de cluster é agrupar objetos semelhantes, faz-se necessária uma medida da distância entre os mesmos. De acordo com Bussab et al. (1990), as medidas de distância são consideradas medidas de dissimilaridades, pois quanto maior a distância, menor a semelhança entre os elementos. Os objetos com menor distância entre si são mais semelhantes, logo são incluídos em um mesmo cluster, enquanto os mais distantes participam de outros agrupamentos.

Para potencializar a utilização de diferentes técnicas para a formação de clusters, Hair Junior et al. (2005), Mingoti (2005) e Fávero et al. (2009) recomendam a utilização dos métodos hierárquico e não hierárquico de forma conjunta, no sentido de contemplar as melhores características de cada um. Desse modo, utilizar-se-á primeiramente o método hierárquico para a definição do número de grupos. Nessa etapa, seguindo a sugestão de Hair Junior et al. (2005), será utilizado o procedimento de Ward, que é o mais recomendado quando se utiliza a matriz de distância quadrática euclidiana. Optou-se pelo método de Ward, pois suas partições buscam minimizar a perda associada a cada agrupamento.

Na sequência, o número de grupos sugeridos pelo método hierárquico será calibrado no método não hierárquico k-means, que é o mais conhecido e utilizado na literatura (Mingoti, 2005). Boa parte dos clusters já converge nos primeiros passos do algoritmo, ficando somente uma quantidade pequena de clusters que ainda se modificam. Assim, a partição final reflete as características de cada elemento agrupado, o que permite que sejam feitas interpretações dos agrupamentos formados e propriedades das variáveis encontradas em cada grupo (Fávero et al., 2009). No sentido de buscar maior robustez na definição da partição final, como sugerem Kassambara (2017), Fávero et al. (2009) e Mingoti (2005), o processo iterativo do k-means se iniciará a partir das sementes iniciais obtidas no método hierárquico.

\subsection{Shift-share}

O shift-share tem por finalidade descrever o crescimento econômico de uma determinada região em termos da sua estrutura produtiva. A partir de um conjunto de identidades contábeis, o método aponta duas razões para o crescimento de uma região: i) a região pode crescer mais que as outras em virtude da sua composição produtiva, ou seja, apresenta, majoritariamente, setores dinâmicos, ou ii) porque a sua estrutura tem participação crescente no total das regiões, independentemente da existência de setores dinâmicos. O crescimento regional, portanto, é decomposto entre um componente estrutural e um componente diferencial (Haddad, 1989).

Esse método analisa o crescimento de determinada variável de interesse, medida em nível regional, considerando os diferentes fatores que podem influenciar o seu 
comportamento (Silva, 2011). Segundo esse autor, as diferenças de crescimento entre as regiões são resultantes não apenas das diferentes estruturas produtivas de cada região, mas também das diversas vantagens de caráter locacional, tais como: custos de transporte e de aquisição de insumos diferenciados, disponibilidade de mão de obra qualificada e capacidade empresarial.

Ao longo do tempo houve diversas propostas de reformulação do método original, no sentido de superar limitações. O presente artigo utiliza a reformulação proposta por Esteban-Maquillas (1972), pois esse autor incorpora os efeitos alocação e competitividade, buscando eliminar a influência do efeito estrutural resultante da distribuição setorial do emprego do ano inicial para o cálculo do efeito diferencial. Dessa forma, pode-se formalizar o modelo levando em conta que o ano inicial é representado por 0 (2010) e o ano final corresponde a 1 (2015). Os componentes do crescimento regional são definidos como: variação regional $(R)$, variação estrutural $(E)$, variação diferencial $(D)$, efeito competitivo (C) e efeito de alocação (A), ou seja:

$$
\begin{aligned}
& \underbrace{\sum_{i} E_{i j}{ }^{1}-\sum_{i} E_{i j}{ }^{0}}_{\text {VT }}=
\end{aligned}
$$

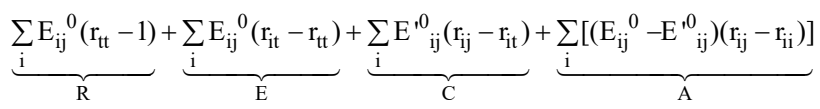

Em que: $\mathrm{r}_{\mathrm{it}}=\left(\sum \sum \mathrm{E}_{\mathrm{ij}}^{1}\right) / \sum \sum \mathrm{E}_{\mathrm{ij}}^{0}=$ crescimento do $\mathrm{VAB}$ de todos os clusters; $\mathrm{r}_{\mathrm{it}}=\sum \mathrm{E}_{\mathrm{ij}}^{1} / \sum \mathrm{E}_{\mathrm{ij}}^{0}=$ taxa de crescimento do VA $A^{i} B^{j}$ no setor $i$ em todos os clusters; $r_{i j}=E_{i j}^{1} / E_{i j}^{0}=$ taxa de crescimento do VAB do setor $i$ do cluster $j ; \mathrm{E}_{\mathrm{ij}}^{\prime}=\sum \mathrm{E}_{\mathrm{ij}}\left(\sum \mathrm{E}_{\mathrm{ij}} / \sum \sum \mathrm{E}_{\mathrm{ij}}\right)=\mathrm{VAB}$ homotético, isto é, o VAB no setor $i$ se o cluster $j$ apresentasse a mesma èstrùtura 'de $\mathrm{VAB}$ do total dos clusters. A principal contribuição de Esteban-Maquillas (1972), portanto, foi adicionar à identidade mais dois componentes ao método original, isto é, o efeito competitivo (C) e o efeito alocação (A).

A variável mais utilizada na literatura para a aplicação do shift-share é o emprego, em razão da ampla disponibilidade dessa variável pelos órgãos estatísticos. No entanto, o método pode ser aplicado para qualquer variável de interesse ${ }^{3}$. Neste artigo são utilizados dados de VAB, em que a VT representa a variação total do VAB setorial entre 2010 e 2015 do cluster $j$. Acredita-se que, nesse caso, o VAB retrata de maneira mais fidedigna a realidade regional, principalmente em detrimento dos dados de emprego da Relação Anual de Informações Sociais (RAIS), uma vez que os mesmos só levam em conta o emprego formal. A Variação Líquida Total (VLT) é dada por:

$\mathrm{VLT}=\mathrm{VT}-\mathrm{R}=\mathrm{E}+\mathrm{C}+\mathrm{A}$

Em que $\mathrm{R}=$ acréscimo de VAB se o cluster $j$ apresentasse a taxa de crescimento do VAB de todos os clusters.

De acordo com a definição do efeito alocação proposto por Esteban-Maquillas (1972), podem-se expressar as classificações possíveis por meio do resultado desse efeito, conforme mostra o Quadro $1^{4}$.

\footnotetext{
${ }^{3}$ Ver, por exemplo, aplicações para VAB industrial per capita (Souza et al., 2018), exportações (Ferreira et al., 2009) e produtividade (Rocha, 2007).

${ }^{4}$ Alguns autores utilizam mais tipologias, ver Gonçalves Júnior \& Galete (2008), por exemplo.
} 
Quadro 1. Resultados do efeito alocação

\begin{tabular}{|c|c|c|c|c|}
\hline & \multirow[b]{2}{*}{ Alternativas } & \multicolumn{3}{|c|}{ Componentes } \\
\hline & & Efeito alocação & Especialização & $\begin{array}{l}\text { Vantagem } \\
\text { competitiva }\end{array}$ \\
\hline II & $\begin{array}{l}\text { Vantagem competitiva, } \\
\text { especializada }\end{array}$ & + & + & + \\
\hline II & $\begin{array}{l}\text { Vantagem competitiva, } \\
\text { não especializada }\end{array}$ & - & - & + \\
\hline III & $\begin{array}{l}\text { Desvantagem competitiva, } \\
\text { não especializada }\end{array}$ & + & - & - \\
\hline IV & $\begin{array}{c}\text { Desvantagem competitiva, } \\
\text { especializada }\end{array}$ & - & + & - \\
\hline
\end{tabular}

Fonte: Haddad (1989).

\subsection{Base de dados}

Para a construção da base de dados foram utilizadas informações provenientes do IBGE, da RAIS, disponibilizadas pelo atual Ministério da Economia, à época Ministério do Trabalho, e do IDH-M obtido no Atlas de Desenvolvimento Humano do Programa das Nações Unidas (PNUD).

Para agrupar os municípios que compõem a região do MATOPIBA utilizaram-se as seguintes variáveis coletadas em nível municipal para o ano de 2015: i) Quociente locacional (QL) da agropecuária; ii) número de estabelecimentos agropecuários (EST); iii) renda média nominal da agropecuária (REN); iv) produtividade agrícola (PROD); v) VAB per capita da agropecuária (VAB); e vi) IDH-M, sendo este referente ao ano de $2010^{5}$. Ainda que de forma arbitrária, a escolha das referidas variáveis reflete características importantes da região de estudo e, portanto, são pertinentes para a aplicação da análise de cluster. Além disso, como será mostrado ao final desta seção, a partir da utilização de estatísticas específicas (Hopkins e "alfa" de (ronbach) a base se mostrou adequada para a análise de cluster.

As variáveis coletadas englobam, em nível detalhado, informações socioeconômicas para classificar os municípios que compõem a região do MATOPIBA. O QL é definido aqui pela razão entre a participação do VAB da agropecuária no VAB total de determinado município $j$ e a participação do VAB da agropecuária no total do VAB total de todos os municípios, ou seja, do MATOPIBA (região de referência), isto é:

$$
\mathrm{QL}_{\mathrm{ij}}=\frac{\mathrm{E}_{\mathrm{ij}} / \mathrm{E}_{\mathrm{i}}}{\mathrm{E}_{\mathrm{j}} / \mathrm{E}_{. .}}
$$

Em que: $E_{\mathrm{ij}}: \operatorname{VAB}$ da agropecuária no munícipio $j$; $\mathrm{E}_{\mathrm{i} . \mathrm{V}} \mathrm{VAB}$ total do município $j$; $\mathrm{E}_{\mathrm{j} j}: \mathrm{VAB}$ da agropecuária do MATOPIBA; e E.: VAB total do MATOPIBA.

O QL mede o grau de especialização do setor agropecuário nos municípios do MATOPIBA. Se o QL > 1 isso sugere que o município é especializado no setor agropecuário. O QL tem sido amplamente utilizado na literatura como proxy de externalidade de especialização (Glaeser et al., 1992; Combes, 2000; Ribeiro et al., 2017).

A produtividade agrícola foi definida como o rendimento da terra das culturas selecionadas, medida em toneladas produzidas por hectare plantado (Costa et al., 2013). Dessa forma, por meio dos dados coletados da Produção Agrícola Municipal (PAM) sobre quantidade produzida e área plantada ou destinada à colheita (hectares) foi possível construir a variável produtividade agrícola para os municípios do MATOPIBA. Vale ressaltar que a ideia

\footnotetext{
${ }^{5}$ Não há dado disponível do IDH-M para 2015. Tentou-se utilizar dados do IFDM da Firjan para 2015, no entanto os mesmos não estavam disponíveis para todos os municípios do MATOPIBA.
} 
é ter uma medida síntese de produtividade agrícola, portanto optou-se em utilizar a variável de forma agregada e não desagregada para diferentes culturas, por exemplo.

As variáveis sobre o número de estabelecimentos e a renda média nominal da agropecuária foram obtidas na RAIS, as quais consideram apenas vínculos formais. Apenas o Censo Demográfico disponibiliza tanto dados formais como informais, porém as informações mais recentes são referentes ao ano de 2010 e podem não retratar a atual realidade dos municípios do MATOPIBA. Apesar disso, as informações da RAIS têm sido utilizadas em estudos recentes (Santos et al., 2018; Garsous et al., 2017; Ribeiro et al., 2017; Ribeiro \& Andrade, 2015; Ribeiro \& Lopes, 2015).

Para o cálculo do shift-share são utilizadas informações do VAB dos grandes setores econômicos (agropecuária, indústria, serviços e administração pública) da base PIB municípios do IBGE nos anos 2010 e 2015. Ressalta-se que o shift-share foi aplicado para os grupos formados pela análise de cluster, ou seja, os valores do VAB setorial dos municípios pertencentes a um mesmo cluster foram somados. Não obstante, todos os valores foram deflacionados a preços de 2015 a partir do deflator implícito do PIB, objetivando valores constantes e, portanto, crescimento real.

Para verificar a adequação da base de dados à análise de cluster, foi utilizada a estatística de Hopkins, a qual, segundo Kassambara (2017, p. 124), pode ser especificada como:

$\mathrm{H}=\frac{\sum_{\mathrm{i}=1}^{\mathrm{n}} \mathrm{y}_{\mathrm{i}}}{\sum_{\mathrm{i}=1}^{\mathrm{n}} \mathrm{x}_{\mathrm{i}}+\sum_{\mathrm{i}=1}^{\mathrm{n}} \mathrm{y}_{\mathrm{i}}}$

Em que: $\mathrm{n}$ é o número de objetos; $\mathrm{y}_{\mathrm{i}}$ é a distância entre $\mathrm{n}$ objetos selecionados aleatoriamente e seus vizinhos mais próximos; e $x_{i}$ é a distância entre $n$ objetos selecionados a partir de uma amostra distribuída uniformemente e seus vizinhos mais próximos.

O valor de $\mathrm{H}$ em torno de 0,5 significa que $\sum^{n} x_{i}$ e $\sum^{n} y_{i}$ são próximos e, portanto, a base de dados é uniformemente distribuída $\left(H_{0}\right)$. A hipótéte difternativa é que a base de dados não é uniformemente distribuída, i.e., apresenta clusters significativos. Em outras palavras, quanto mais próximo de zero o valor de $\mathrm{H}$, maior a tendência à clusterização. $\mathrm{O}$ valor da estatística de Hopkins foi de 0,0945 , que está próximo de zero, o que indica que a base de dados é adequada para a análise de cluster.

Somado a isso, foi calculado o coeficiente "alfa" de Cronbach (Cronbach, 1951) no intuito de verificar a confiabilidade da base de dados em relação à sua consistência interna. Sua especificação é dada por:

$\alpha=\frac{\mathrm{n}}{\mathrm{n}-1}\left(1-\frac{\sum_{\mathrm{i}} \mathrm{V}_{\mathrm{i}}}{\mathrm{V}_{\mathrm{t}}}\right)$

Em que: $n$ é o número de itens; $v_{t}$ é a variância dos escores dos testes; e $v_{i}$ é a variância dos escores dos itens após ponderação.

O coeficiente de Cronbach varia entre 0 e 1 e mede o grau de covariância de uma série de itens. Quanto mais próximo de um, maior a confiabilidade da escala. De acordo com Hair Junior et al. (2005), o valor de 0,6 é o mínimo aceitável para o referido coeficiente. Para o presente trabalho, o valor do coeficiente "alfa" de Cronbach foi de 0,651, o que revela, portanto, confiabilidade da base de dados utilizada.

\section{Resultados e discussões}

No intuito de melhorar a organização da exposição desta seção, inicialmente apresentam-se as estatísticas descritivas da base de dados e na sequência os resultados da análise de cluster e do shift-share. A Tabela 2 apresenta as estatísticas descritivas das seis variáveis utilizadas na análise de cluster considerando todos os 337 municípios do MATOPIBA. 
Tabela 2: Estatísticas descritivas da base de dados

\begin{tabular}{ccccc} 
Variáveis & Média & Desvio- padrão & Mínimo & Máximo \\
QL & 1,22 & 0,76 & 0 & 3,60 \\
EST & 34 & 59 & 0 & 405 \\
REN & $1.130,3$ & 449,8 & 0 & $3.075,0$ \\
PROD & 5,1 & 6,4 & 0,4 & 60,0 \\
IDH-M & 0,61 & 0,05 & 0,44 & 0,79 \\
VAB & 3.965 & 6.969 & 100 & 64.250 \\
\hline
\end{tabular}

Fonte: Elaboração própria com base no SPSS.

A partir da Tabela 2, percebe-se forte heterogeneidade entre os municípios do MATOPIBA quando se consideram, principalmente, variáveis como número de estabelecimentos agropecuários (EST), produtividade agrícola (PROD) e VAB per capita da agropecuária, uma vez que o desvio-padrão dessas variáveis foi superior à respectiva média. O comportamento mais homogêneo entre as variáveis foi o do IDH-M que, em média, foi de 0,61 . Os municípios do MATOPIBA apresentam em média um $\mathrm{QL}=1,22$ e, como esperado, podem ser considerados especializados na agropecuária. O número de estabelecimentos da agropecuária e o rendimento médio do setor foram de 34 e $R \$ 1.130,30$, respectivamente, ao passo que a produtividade agrícola e o VAB per capita da agropecuária foram na média de 5,1 toneladas por hectare (t/ha) e $R \$ 3.695$, respectivamente.

No que se refere à análise de cluster, os primeiros passos foram a padronização das variáveis ${ }^{6}$ e a definição do número de grupos. Para tanto, utilizou-se, num primeiro momento, o método hierárquico com base no procedimento de Ward e na distância quadrática euclidiana. Hair Junior et al. (2005), Mingoti (2005) e Fávero et al. (2009) afirmam que não existe uma regra de parada objetiva para determinar o número de clusters. No entanto, existem algumas estatísticas que podem auxiliar o pesquisador nessa determinação. Assim, buscando maior robustez, a definição do número de clusters foi baseada conjuntamente no dendograma (Figura 1) e na estatística pseudo T², proposta por Duda \& Hart (1973).

A partir da Figura 3 foi traçada uma reta horizontal de cor preta no local em que se observa maior ramificação da quantidade de grupos. A quantidade de linhas verticais que a linha horizontal cruza (diferentes cores) sugere a quantidade ideal de clusters que, para esse estudo, foi claramente cinco.

\footnotetext{
${ }^{6}$ Para padronizar as variáveis, foi utilizada a seguinte fórmula: $Z=\frac{X-\mu}{\sigma}$; em que: $\mu$ e $\sigma$ é a média e o desvio-padrão
} da variável X, respectivamente. 


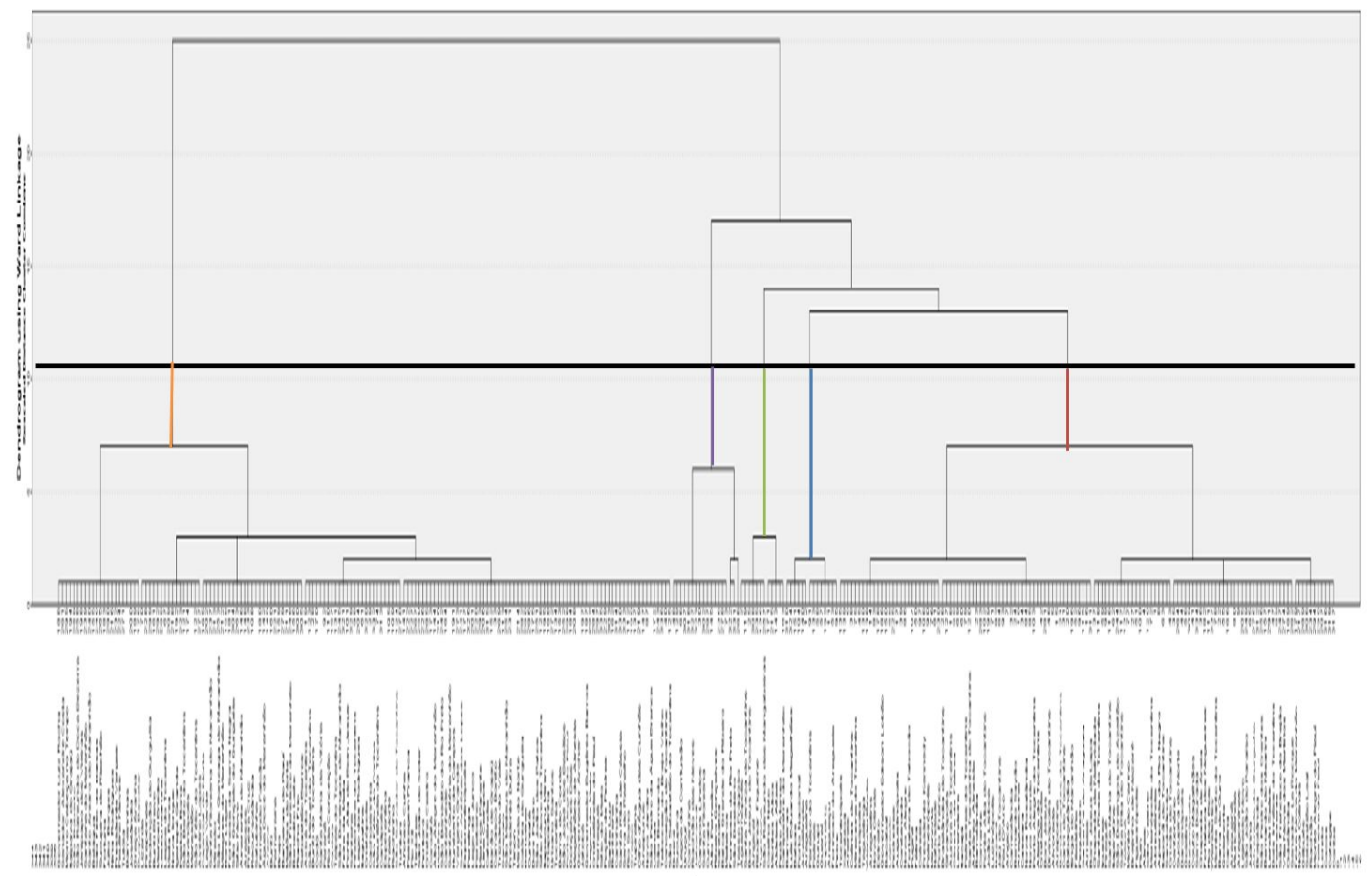

Figura 3: Dendograma. Fonte: Elaboração própria a partir do software SPSS.

Dada a possível subjetividade da análise do dendograma, optou-se também em utilizar conjuntamente a estatística pseudo $\mathrm{T}^{2}$ (Duda \& Hart, 1973), conforme mostra a Tabela 3. De acordo com Mingoti (2005), o número de clusters utilizados na partição final corresponderá ao passo em que se observa o valor máximo da estatística pseudo $\mathrm{T}^{2}$ ou aquele imediatamente anterior. A partir da Tabela 1 o ponto máximo da estatística $\mathrm{T}^{2}(87,77)$ é obtido no passo com cinco grupos, que coincide com o resultado sugerido pelo dendograma (Figura 3) e que, portanto, será adotado neste trabalho.

Tabela 3: Estatística pseudo $\mathrm{T}^{2}$

\begin{tabular}{cccccc}
$\begin{array}{c}\mathbf{N}^{\circ} \text { de } \\
\text { clusters }\end{array}$ & Pseudo $\mathbf{T}^{2}$ & $\begin{array}{c}\mathbf{N}^{\circ} \text { de } \\
\text { clusters }\end{array}$ & Pseudo $\mathbf{T}^{2}$ & $\begin{array}{c}\mathbf{N}^{\circ} \text { de } \\
\text { clusters }\end{array}$ & ${\text { Pseudo } \mathbf{T}^{2}}^{2}$ \\
\hline 1 & 86,59 & 11 & 22,51 & 21 & 14,72 \\
2 & 38,01 & 12 & 30,68 & 22 & 29,62 \\
3 & 46,8 & 13 & 34,17 & 23 & 20,35 \\
4 & 76,01 & 14 & 13,71 & 24 & 5,6 \\
5 & 87,77 & 15 & 2,51 & 25 & 7,94 \\
6 & 56,1 & 16 & 18,75 & 26 &. \\
7 & 18,78 & 17 & 20,08 & 27 & 11,98 \\
8 & 43,02 & 18 & 18,42 & 28 & 10,97 \\
9 & 11,75 & 19 & 2,79 & 29 & 7,56 \\
10 & 45,58 & 20 & 6,13 & 30 & 3,46 \\
\hline
\end{tabular}

Fonte: Elaboração própria com base no STATA.

Após a definição do número de grupos e das sementes iniciais pelo método hierárquico, utilizou-se o método não hierárquico, em particular o método K-Means de aglomeração, com o objetivo de gerar os cinco agrupamentos. 
Para corroborar a consistência da partição final foi também realizada uma análise discriminante linear de Fischer (Mingoti, 2005), de tal modo que a partição final apresentou uma probabilidade de erro de classificação abaixo de $5 \%$. De posse dessa informação, a Tabela 4 apresenta os resultados dos cinco grupos formados.

Tabela 4: Resultado dos clusters dos municípios do MATOPIBA

\begin{tabular}{|c|c|c|c|c|c|c|}
\hline Variável & Cluster & $\mathrm{N}^{\circ}$ de municípios & Média & Desvio-padrão & Mínimo & Máximo \\
\hline \multirow{6}{*}{ QL } & 1 & 132 & 1,51 & 0,66 & 0,10 & 2,90 \\
\hline & 2 & 16 & 0,68 & 0,68 & 0,00 & 2,20 \\
\hline & 3 & 8 & 1,60 & 0,64 & 0,70 & 2,50 \\
\hline & 4 & 17 & 3,07 & 0,40 & 2,20 & 3,60 \\
\hline & 5 & 164 & 0,83 & 0,39 & 0,10 & 1,80 \\
\hline & Total & 337 & 1,22 & 0,76 & 0,00 & 3,60 \\
\hline \multirow{6}{*}{ EST } & 1 & 132 & 34 & 26 & 1 & 137 \\
\hline & 2 & 16 & 228 & 88 & 124 & 392 \\
\hline & 3 & 8 & 21 & 20 & 3 & 66 \\
\hline & 4 & 17 & 95 & 102 & 21 & 405 \\
\hline & 5 & 164 & 10 & 14 & 0 & 100 \\
\hline & Total & 337 & 34 & 59 & 0 & 405 \\
\hline \multirow{6}{*}{$\begin{array}{c}\text { REN (em } \\
\text { R\$) }\end{array}$} & 1 & 132 & $1.344,95$ & 327,50 & 740,60 & $3.075,00$ \\
\hline & 2 & 16 & $1.380,09$ & 255,24 & 991,05 & $1.800,59$ \\
\hline & 3 & 8 & $1.317,43$ & 358,32 & 856,02 & $1.828,47$ \\
\hline & 4 & 17 & $1.829,85$ & 270,79 & $1.342,43$ & $2.276,60$ \\
\hline & 5 & 164 & 851,61 & 371,25 & 0,00 & $1.467,38$ \\
\hline & Total & 337 & $1.130,34$ & 449,81 & 0,00 & $3.075,00$ \\
\hline \multirow{6}{*}{$\begin{array}{l}\text { PROD } \\
\text { (em } \\
\text { t/ha) }\end{array}$} & 1 & 132 & 4,94 & 3,49 & 0,65 & 20,71 \\
\hline & 2 & 16 & 4,19 & 2,08 & 2,25 & 10,50 \\
\hline & 3 & 8 & 39,72 & 12,75 & 25,00 & 59,99 \\
\hline & 4 & 17 & 3,65 & 0,77 & 2,82 & 5,71 \\
\hline & 5 & 164 & 3,70 & 2,65 & 0,35 & 15,52 \\
\hline & Total & 337 & 5,06 & 6,44 & 0,35 & 59,99 \\
\hline \multirow{6}{*}{ IDH-M } & 1 & 132 & 0,63 & 0,04 & 0,53 & 0,71 \\
\hline & 2 & 16 & 0,72 & 0,04 & 0,67 & 0,79 \\
\hline & 3 & 8 & 0,62 & 0,07 & 0,51 & 0,73 \\
\hline & 4 & 17 & 0,60 & 0,04 & 0,54 & 0,68 \\
\hline & 5 & 164 & 0,58 & 0,04 & 0,44 & 0,68 \\
\hline & Total & 337 & 0,61 & 0,05 & 0,44 & 0,79 \\
\hline \multirow{6}{*}{$\begin{array}{c}\mathrm{VAB}(\mathrm{em} \\
\mathrm{R} \$)\end{array}$} & 1 & 132 & 4.394 & 3.087 & 500 & 17.160 \\
\hline & 2 & 16 & 3.679 & 3.701 & 170 & 10.200 \\
\hline & 3 & 8 & 4.509 & 3.841 & 980 & 11.380 \\
\hline & 4 & 17 & 27.531 & 15.720 & 15.530 & 64.250 \\
\hline & 5 & 164 & 1.179 & 640 & 100 & 3.850 \\
\hline & Total & 337 & 3.965 & 6.969 & 100 & 64.250 \\
\hline
\end{tabular}

Fonte: Elaboração própria com base no SPSS.

O cluster 1 é composto por 132 municípios (39,2\%). Em média, há nesse grupo 34 estabelecimentos agropecuários com remuneração média de $R \$ 1.344,95$, o que pode ter contribuído, em parte, para esse grupo ser especializado na agropecuária $(\mathrm{QL}=1,51)$. 
Sua produtividade agrícola foi de 4,94 t/ha e apresentou o segundo maior VAB per capita da agropecuária ( $R \$ 4.394)$ entre os grupos.

O cluster 2 é composto por 16 municípios (4,7\%), entre eles: Paraíso do Tocantins/TO, Açailândia/MA, Balsas/MA, Imperatriz/MA, Barreiras/BA e Luís Eduardo Magalhães/BA. O cluster reúne 8 dos 10 maiores municípios em termos de PIB total, dentre eles destacam-se no estado do Maranhão Imperatriz e Açailândia, que possuem uns dos maiores PIB no estado e relevante participação no setor industrial. Enquanto os municípios de Luiz Eduardo Magalhães, Barreiras e Balsas, por sua vez, destacam-se no setor agrícola com a produção de grãos, ainda que boa parte do VAB total desses municípios seja gerada pelo setor de serviços. Como consequência disso, o QL da agropecuária foi o menor entre os agrupamentos $(0,68)$, apresentou em média a produtividade agrícola de 4,19 t/ha e um dos menores VAB per capita da agropecuária ( $R \$ 3.679)$. Por outro lado, o cluster 2 possui, em média, o maior número de estabelecimentos agrícolas (228) e detém o maior IDH-M $(0,72)$. Como obervado por Porcionato et al. (2018), alguns desses municípios estão localizados em microrregiões onde houve maior aumento de IDH-M comparando 2000 e 2010.

O cluster 3 é formado por apenas 8 municípios (menor grupo), são eles: Pedro Afonso/TO, Santa Tereza do Tocantins/TO, Aldeias Altas/MA, Campestre do Maranhão/MA, Coelho Neto/MA, Montes Altos/MA, Ribamar Fiquene/MA e São Raimundo das Mangabeiras/MA. Ainda que, em média, esse grupo tenha apenas 21 estabelecimentos agropecuários e remuneração média do setor de $R \$ 1.317,43$, ele pode ser considerado especializado na agropecuária uma vez que $\circ \mathrm{QL}=1,60$. Além disso, a produtividade agrícola de $39,72 \mathrm{t} / \mathrm{ha}$ foi a maior entre os clusters formados, aproximadamente 8 vezes o valor da média dos clusters, ao passo que o VAB agropecuário per capita foi de $\mathrm{R} \$ 4.509$ (o segundo maior). É importante destacar que esses 8 municípios apresentam as maiores produtividades agrícolas do MATOPIBA, o que resultou numa média bem destoante dos demais grupos.

O cluster 4, com 17 municípios, foi o mais especializado no setor agropecuário (QL =3,07). Os municípios Tasso Fragoso/MA, Formosa do Rio Preto/BA e São Desidério/BA são destaque nacional no cultivo da soja, segundo Pereira et al. (2018). Apenas Formosa do Rio Preto produziu $11 \%$ de toda a produção do MATOPIBA, seguida de São Desidério, que produziu 8,3\%, para o ano de 2014. Esse grupo apresenta, ainda, a maior renda média da agropecuária $(R \$ 1.829,85)$, o maior $\operatorname{VAB}(\mathrm{R} \$ 27.531)$ e apresenta o segundo maior número de estabelecimentos agrícolas (95). Em contraste com esses resultados, apresentou o menor valor da produtividade agrícola (3,65 t/ha). Além disso, apresentou em média o segundo menor IDH-M $(0,60)$, que ficou abaixo do da região como um todo $(0,61)$. Ainda que esse grupo tenha apresentado o melhor desempenho da agropecuária medido, por exemplo, pela renda média, VAB e QL, O IDH-M sugere que essa riqueza não se refletiu em maiores níveis de desenvolvimento. Não obstante, o resultado da produtividade agrícola deve ser interpretado com cautela, uma vez que se utiliza uma medida agregada de produtividade.

O cluster 5 apresenta 165 municípios e, da mesma forma que o cluster 2, foram os únicos grupos não especializados na agropecuária $(\mathrm{QL}=0,83)$. Esse grupo apresenta o menor número de estabelecimentos agropecuários (10) e a remuneração média do setor mais baixa entre os grupos ( $R \$ 851,61)$, a única abaixo da média do MATOPIBA ( $R \$ 1.130,34)$. Isso, de certo modo, pode ter se refletido na produtividade agrícola de 3,70 t/ha (segunda menor) e no VAB agropecuária per capita que foi o menor entre os grupos ( $R \$ 1.179)$ e bem abaixo da média regional ( $R \$ 3.965)$. Contudo, 3 de seus municípios estão entre os 10 maiores VAB de administração: Caxias, Timon e Codó, todos situados no Maranhão. Além disso, os 2 primeiros se destacam entre os 10 maiores municípios em número de estabelecimentos empresariais e outras organizações, de acordo com análise de Buainain et al. (2017), em análise dos anos 2010 e 2012. Na análise do cluster, a informação mais preocupante é a média para o IDH-M de 0,58, abaixo do padrão de desenvolvimento do MATOPIBA $(0,61)$. Dos 164 municípios, 103 deles encontram-se no Maranhão, estado que possui a maior concentração de favorecidos do Benefício de Prestação Continuada (BPC), de acordo com Porcionato et al. (2018); entre as 10 microrregiões que mais possuem beneficiários estão: Médio Mearim ( $\left.1^{\circ}\right)$, Caxias $\left(3^{\circ}\right)$, Codó $\left(4^{\circ}\right)$ e Alto Mearim e Grajaú $\left(6^{\circ}\right)$ no Maranhão, que encontram-se nesse cluster. 
A Figura 4 mostra a distribuição espacial dos clusters do MATOPIBA. Nota-se que a maior parte dos municípios do cluster 5 está localizada ao extremo norte ou em área de fronteira, a qual, de certo modo, pode ser considerada a área menos desenvolvida, já que apresentou o IDH-M, em média, de 0,58. Os municípios mais desenvolvidos (cluster 2) e os mais pujantes do ponto de vista da atividade agrícola (cluster 3 ) localizam-se na parte mais central e no extremo oeste da região.

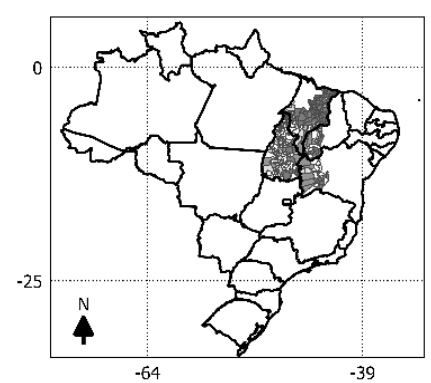

Legenda

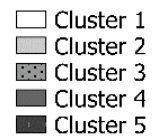

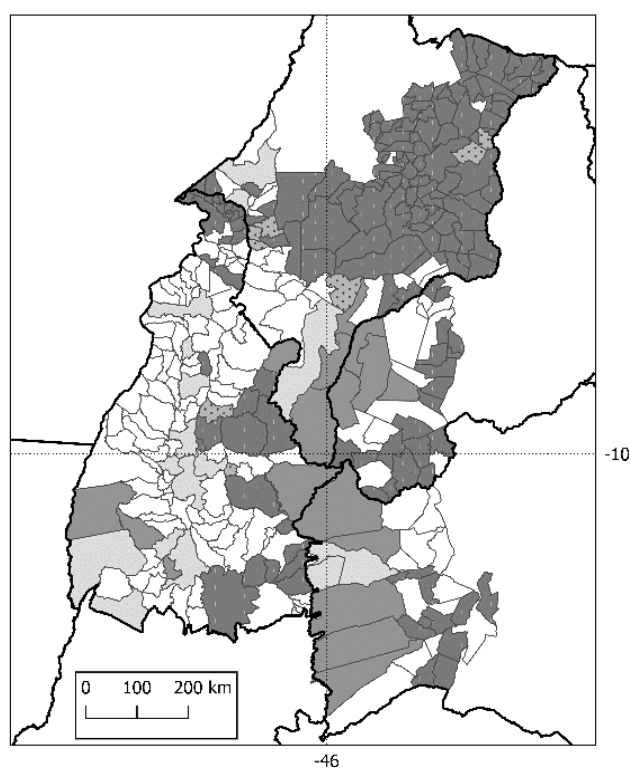

Figura 4: Distribuição espacial dos clusters - municípios do MATOPIBA. Fonte: Elaboração própria.

No intuito de complementar a análise de cluster, propõe-se a partir do shift-share identificar, inicialmente, em quais setores os cinco grupos formados apresentam vantagens competitivas e de especialização. O Quadro 2 apresenta a decomposição do efeito alocação por setor produtivo do MATOPIBA, considerando o crescimento real do VAB entre 2010 e 2015. Essa análise permite verificar se o MATOPIBA está especializado nos setores em que apresenta maiores vantagens competitivas ou não (Simões, 2006) a partir das quatro tipologias apresentadas no Quadro 1.

Quadro 2: Decomposição do efeito alocação por setor produtivo do MATOPIBA, 2010-2015

\begin{tabular}{|c|c|c|c|c|}
\hline Cluster & Agropecuária & Indústria & Serviços & $\begin{array}{l}\text { Administração } \\
\text { pública }\end{array}$ \\
\hline 1 & $\begin{array}{l}\text { Desvantagem } \\
\text { competitiva, } \\
\text { especializado }\end{array}$ & $\begin{array}{c}\text { Desvantagem } \\
\text { competitiva, não } \\
\text { especializado }\end{array}$ & $\begin{array}{c}\text { Vantagem } \\
\text { competitiva, não } \\
\text { especializado }\end{array}$ & $\begin{array}{l}\text { Desvantagem } \\
\text { competitiva, } \\
\text { especializado }\end{array}$ \\
\hline 2 & $\begin{array}{c}\text { Vantagem } \\
\text { competitiva, não } \\
\text { especializado }\end{array}$ & $\begin{array}{l}\text { Desvantagem } \\
\text { competitiva, } \\
\text { especializado }\end{array}$ & $\begin{array}{l}\text { Desvantagem } \\
\text { competitiva, } \\
\text { especializado }\end{array}$ & $\begin{array}{l}\text { Vantagem competitiva, } \\
\text { não especializado }\end{array}$ \\
\hline 3 & $\begin{array}{c}\text { Vantagem } \\
\text { competitiva, } \\
\text { especializado }\end{array}$ & $\begin{array}{c}\text { Vantagem } \\
\text { competitiva, não } \\
\text { especializado }\end{array}$ & $\begin{array}{c}\text { Vantagem } \\
\text { competitiva, não } \\
\text { especializado }\end{array}$ & $\begin{array}{c}\text { Vantagem competitiva, } \\
\text { especializado }\end{array}$ \\
\hline 4 & $\begin{array}{l}\text { Vantagem } \\
\text { competitiva, } \\
\text { especializado }\end{array}$ & $\begin{array}{c}\text { Vantagem } \\
\text { competitiva, não } \\
\text { especializado }\end{array}$ & $\begin{array}{c}\text { Vantagem } \\
\text { competitiva, não } \\
\text { especializado }\end{array}$ & $\begin{array}{l}\text { Vantagem competitiva, } \\
\text { não especializado }\end{array}$ \\
\hline 5 & $\begin{array}{l}\text { Desvantagem } \\
\text { competitiva, não } \\
\text { especializado }\end{array}$ & $\begin{array}{c}\text { Vantagem } \\
\text { competitiva, não } \\
\text { especializado }\end{array}$ & $\begin{array}{l}\text { Desvantagem } \\
\text { competitiva, não } \\
\text { especializado }\end{array}$ & $\begin{array}{l}\text { Desvantagem } \\
\text { competitiva, } \\
\text { especializado }\end{array}$ \\
\hline
\end{tabular}

Fonte: Elaboração própria. 
Dos quatros setores apresentados no Quadro 2, a agropecuária apresentou o maior número de clusters com vantagem competitiva (2, 3 e 4), sendo que os clusters 3 e 4 são especializados no setor (já evidenciado pela análise de cluster). Isso reforça, portanto, a análise apresentada por meio da Tabela 4 em que o cluster 3, além de ser especializado na agropecuária (QL > 1), apresentou a maior produtividade agrícola. O cluster 4, por sua vez, apresentou o melhor desempenho da agropecuária medido pelo QL, renda média e VAB.

Esses resultados convergem para o já apontado na literatura, ou seja, o MATOPIBA como a nova fronteira com maior potencial para expansão agropecuária brasileira, entretanto, de forma heterogênea devido aos solos, topografia, clima, entre outros fatores estruturais (Buainain et al., 2017). Essa heterogeneidade pode explicar o resultado dos clusters 1 e 5, ou seja, a agropecuária do cluster 1 foi especializada (já apontado pela análise de cluster, QL > 1), mas não apresentou vantagem competitiva. O cluster 5 , por outro lado, foi considerado pela análise anterior o mais incipiente, o que é corroborado pela decomposição do efeito alocação, uma vez que a agropecuária não foi especializada nem competitiva.

O Quadro 2 revela que embora os clusters 3, 4 e 5 apresentem vantagens competitivas para a indústria, a mesma não foi especializada. Interessante notar que este setor só foi especializado no cluster 2, ainda que sem vantagem competitiva. Como apontado na análise anterior, o cluster 2 foi o mais desenvolvido (maior IDH-M) e tem 2 municípios que se destacam no setor industrial, o que pode explicar, em parte, tal resultado.

No que remete ao setor de serviços, os clusters 1, 3 e 4 apresentaram vantagem competitiva, mas não foram especializados. Os clusters 2 e 5, por outro lado, não apresentaram vantagens competitivas, mas o cluster 2 foi especializado. Um fato interessante é que os clusters 3 e 4 foram especializados e também apresentaram vantagem competitiva na agropecuária, ou seja, percebe-se uma complementariedade entre as duas atividades. Isso pode ser reflexo do aumento da renda gerada pelo agronegócio, que dinamizou o mercado de trabalho local e aqueceu o setor terciário (Serigati et al., 2017), o qual é relacionado com diversas atividades da agropecuária, como logística, comércio, transporte, entre outras (Porcionato et al., 2018).

A administração pública foi o único setor, além da agropecuária, a apresentar algum cluster (3) com vantagem competitiva e especializado. Os clusters 2 e 4 possuem vantagem competitiva, mas não são especializados. Isso pode ser reflexo de um problema que ocorre na maioria dos municípios brasileiros de pequeno porte, os quais não têm autonomia fiscal e dependem exclusivamente dos repasses estaduais e federais (Marinho \& Jorge, 2015). Espera-se que com a maturação dos investimentos que estão sendo feitos na agropecuária local, os municípios passem a depender menos do setor público (Buainain et al., 2017), visto que não é um setor dinamizador.

Além dos resultados apresentados no Quadro 2, apresenta-se a decomposição total do shift-share. A Variação Líquida Total (VLT) é dada pela soma dos três componentes especificados na Equação 2, isto é, estrutural (E), competitivo (C) e alocação (A). A VLT positiva indica que haveria crescimento medido pelo VAB entre o período inicial e o final (Galete, 2008). Os clusters 3 e 4 do MATOPIBA apresentariam crescimento de VAB, enquanto os clusters 1, 2 e 5 apresentariam VLT negativa, ou seja, deixariam de crescer entre 2010 e 2015 por terem seu crescimento efetivo (medido pelo VAB) menor do que seu crescimento homotético (Haddad, 1989). Dado o escopo deste artigo, a Tabela 5 apresenta a decomposição completa somente para o setor Aaropecuária.

Tabela 5: Resultado da decomposição do shift-share para a agropecuária

\begin{tabular}{ccccc} 
Clusters & VLT (VT - R) & Estrutural (E) & Competitivo (C) & Alocação (A) \\
\hline 1 & 105.111 & 317.272 & -154.190 & -57.971 \\
2 & 347.036 & 216.194 & 300.460 & -169.619 \\
3 & 110.718 & 34.205 & 48.608 & 27.904 \\
4 & 1.766 .432 & 351.249 & 447.210 & 967.973 \\
5 & -1.083 .876 & 326.500 & -1.665 .882 & 255.506 \\
\hline
\end{tabular}

Fonte: Elaboração própria. 
Percebe-se que somente o cluster 5 apresentou VLT negativa para a agropecuária, isto é, seu crescimento efetivo de VAB foi menor do que seu crescimento homotético. Ainda que o efeito estrutural e o efeito alocação sejam positivos, o componente competitivo implicou uma VLT negativa. Isso é perfeitamente coerente com os resultados apresentados anteriormente, em que tal grupo foi apontado como o mais incipiente do ponto de vista da agropecuária. Os demais clusters apresentaram VLT positiva, mas por razões distintas.

A partir do componente estrutural é possível perceber o quanto a composição setorial do MATOPIBA acarretou no aumento ou diminuição de VAB (Ribeiro \& Lopes, 2015). Todos os clusters apresentaram o componente estrutural positivo, ou seja, eles apresentaram a agropecuária dinâmica no período analisado e, portanto, capaz de alavancar esse crescimento acima da média regional (Castilho, 2015).

Os clusters 2, 3 e 4 apresentaram efeito competitivo positivo, o que indica que nesses grupos a taxa de crescimento do VAB da agropecuária foi maior do que a taxa de crescimento desse setor do MATOPIBA. Por outro lado, nos clusters 1 e 5 o efeito competitivo foi negativo. O ganho e/ou perda de competitividade, segundo Haddad (1989), são decorrentes de fatores locacionais como, por exemplo, variações nos custos de transporte, diferencial regional de preços relativos de insumos e incentivos fiscais. O efeito alocação, por sua vez, mostra se os clusters estão especializados nos setores que apresentam vantagens competitivas (ver Quadro 2). Nesse caso, os clusters 1 e 2 apresentaram efeito alocação positivo, ao passo que os clusters 3, 4 e 5 tiveram tal efeito negativo.

\section{Conclusões}

Nos últimos anos, a região do MATOPIBA tem adquirido relevância como fronteira agrícola nacional. No entanto, alguns estudos indicaram a existência de forte concentração intrarregional de renda. Assim, este artigo buscou classificar os 337 municípios do MATOPIBA em grupos a partir de variáveis socioeconômicas para a região, bem como decompor o crescimento setorial dos grupos formados entre 2010 e 2015. Para tanto, foram utilizadas, conjuntamente, a análise de cluster e o shift-share.

Os principais resultados mostraram a formação de cinco clusters no MATOPIBA. Três deles (1, 3 e 4) foram especializados na agropecuária. Por outro lado, o cluster 5 foi considerado o mais incipiente entre os grupos formados, pois além de apresentar os mais baixos indicadores para as variáveis relacionadas à agropecuária, também teve o menor IDH-M e, de acordo com a literatura, apresenta municípios que dependem consideravelmente de programas sociais.

Os resultados do shift-share mostraram-se coerentes com os resultados apresentados pela análise de cluster. O cluster 5 foi o único a apresentar a VLT negativa para a agropecuária, ou seja, deixou de crescer por ter seu crescimento efetivo menor do que seu crescimento homotético, em razão de não apresentar vantagens competitivas para o setor. Os demais grupos apresentaram VLT positiva, mas por razões distintas.

Do ponto de vista de implicações de políticas públicas, sugerem-se políticas baseadas em desconcentração de renda, principalmente nos municípios localizados na área norte do MATOPIBA, ou que sejam direcionadas para os pequenos produtores rurais com estímulo à agricultura familiar ou ao setor de serviços, para que possa se estruturar melhor e dar suporte ao transbordamento da agricultura. Além disso, tais políticas também podem incentivar ganhos de produtividade agrícola na região, uma vez que os municípios dessa área apresentaram, em média, o menor indicador.

Para trabalhos futuros, pretende-se avaliar as possíveis causas desse processo de concentração de renda no MATOPIBA. Outro ponto interessante é saber como encontra-se o padrão das ocupações e respectivas remunerações. Quais mercados se destacam no transbordamento e se existem políticas públicas específicas para a região. Ou seja, aprofundar as investigações acerca dos fatores socioeconômicos que contribuíram para formação do desenvolvimento da região ao longo do tempo. 


\section{Agradecimentos}

Os autores agradecem o apoio financeiro do Conselho Nacional de Desenvolvimento Científico e Tecnológico (CNPq), da Coordenação de Aperfeiçoamento de Pessoal de Nível Superior (CAPES) e da Fundação de Apoio à Pesquisa e a Inovação Tecnológica do Estado de Sergipe (FAPITEC) para a realização desta pesquisa.

\section{Referências}

Alves, E., Souza, G. S., \& Miranda, E. E. (2015). Renda e pobreza rural na região do MATOPIBA (Nota Técnica, No. 10). EMBRAPA. Recuperado em 15 de maio de 2018, de https://www.embrapa.br/gite/publicacoes/NT10_RendaPobrezaMATOPIBA.pdf

Brasil. Ministério da Agricultura, Pecuária e Abastecimento. (2015, novembro 12). Portaria n 244, de 12 de novembro de 2015. Diário Oficial [da] República Federativa do Brasil, Brasília. Recuperado em 01 de agosto de 2019, de https://www.jusbrasil.com.br/diarios/104178828/dou-secao-1-13-11-2015pg-8.

Bresser-Pereira, L. C. (2004). O conceito de desenvolvimento do ISEB rediscutido. Dados, 47(1), 49-84.

Buainain, A. M., Garcia, J. R., \& Vieira Filho, J. E. R. (2017). Dinâmica da economia e da agropecuária no Matopiba (Texto para Discussão, No. 2283). Rio de Janeiro: IPEA.

Bussab, W. O., Miazaki, E. S., \& Andrade, D. (1990). Introdução à análise de agrupamentos (105 p.). São Paulo: Associação Brasileira de Estatística.

Castilho, A. L. (2015). O crescimento econômico da região metropolitana de Curitiba e seu dinamismo entre 2000 e 2010 (Monografia). Curso de Ciências Econômicas, Universidade Federal do Paraná, Curitiba.

Combes, P. P. (2000). Economic structure and local growth: France, 1984-1993. Journal of Urban Economics, 47(3), 329-355.

Costa, L. V., Gomes, M. F. M., Lirio, V. S., \& Braga, M. J. (2013). Produtividade agrícola e segurança alimentar dos domicílios das regiões metropolitanas brasileiras. Revista de Economia e Sociologia Rural, 51(4), 661-680.

Cronbach, L. (1951). Coefficient alpha and the internal structure of tests. Psychometrika, 16(3), 297-334.

Duda, R. O., \& Hart, P. E. (1973). Pattern classification ans scene analysis. New York: John Wiley \& Sons.

Esteban-Maquillas, J. M. (1972). Shift and share analysis revisited. Regional and Urban Economics, 2(3), 249-261.

Everitt, B. S., Landau, S., \& Leese, M. (2001). Cluster analysis (4th ed., 207 p.). London: Arnold.

Fávero, L. P., Belfiore, P., Silva, F. L., \& Chan, B. L. (2009). Análise de dados: modelagem multivariada para tomada de decisões (646 p.). Rio de Janeiro: Elsevier.

Ferreira, M. A. S., Teixeira, E. C., \& Souza, M. C. (2009). Determinantes da receita de exportação brasileira de açúcar e álcool. Revista de Economia Agrícola, 56(2), 47-59.

Galete, R. A. (2008). Uma aplicação do método estrutural-diferencial modificado para a microrregião de Maringá (PR) frente à economia paranaense no período de 1994 a 2008. Revista Estudos do CEPE, 35 55-92.

Garsous, G., Corderi, D., Velasco, M., \& Colombo, A. (2017). Tax incentives and job creation in the tourism sector of Brazil's SUDENE area. World Development, 96, 87-101.

Glaeser, E. L., Kallal, H. D., Scheinkman, J. A., \& Shleifer, A. (1992). Growth in cities. Journal of Political Economy, 100(6), 1126-1152.

Gonçalves Júnior, C. A., \& Galete, R. A. (2008). O método estrutural-diferencial: aplicação da adaptação de Herzog e Olsen para a microrregião de Maringá frente à economia paranaense 1994/2008. Informe Gepec, 14(2), 149-165.

Haddad, P. R. (Ed.). (1989). Economia regional, teorias e métodos de análise. Fortaleza: BNB/ ETENE.

Hair Junior, F. F., Anderson, R. E., Tathan, R. L., \& Black, W. C. (2005). Análise multivariada de dados. Bookman: Porto Alegre.

Horvat, R., Watanabe, M., \& Yamaguchi, C. K. (2015). Fertilizer consumption in the region MATOPIBA and their reflections on Brazilian soybean production. International Journal of Agriculture and Forestry, 5(1), 52-59.

Instituto Brasileiro de Geografia e Estatística - IBGE. (2017). Sistema de Contas Regionais: Brasil: 2015. Rio de Janeiro: IBGE. 
Kassambara, A. (2017). Practical guide to cluster analysis in R. 1st ed. STHDA.

Mardia, A. K. V., Kent, J. T., \& Bibby, J. M. (1997). Multivariate analysis (518 p.). London: Academic Press.

Marinho, A. P. S., \& Jorge, M. A. (2015). O planejamento local é mais eficiente? Uma análise de 14 municípios sergipanos de pequeno porte. Nova Economia, 25(1), 123-142.

Mingoti, S. A. (2005). Análise de dados através de métodos de estatística multivariada: uma abordagem aplicada. Belo Horizonte: Editora UFMG.

Miranda, E. E., Magalhães, L. A., \& Carvalho, C. A. (2014). Nota técnica $n^{\circ}$ 1: proposta de delimitação territorial do MATOPIBA. Embrapa. Recuperado em 20 de abril de 2016, de https://www.embrapa.br/gite/publicacoes/NT1_DelimitacaoMatopiba.pdf

Pereira, C. N., Castro, C. N., \& Porcionato, G. L. (2018). Dinâmica econômica, infraestrutura e logística no Matopiba (Texto para Discussão, No. 2382). Brasília: IPEA.

Porcionato, G. L., Castro, C. N., \& Pereira, C. N. (2018). Aspectos sociais do MATOPIBA: análise sobre o desenvolvimento humano e a vulnerabilidade social (Texto para Discussão, No. 2387). Rio de Janeiro: IPEA.

Rezende, G. C. (2006). Políticas trabalhista, fundiária e de crédito agrícola no Brasil: uma avaliação crítica. Revista de Economia e Sociologia Rural, 44(1), 47-78.

Ribeiro, L. C. S., \& Andrade, J. R. L. (2015). Characterization of tourism clusters in Brazil. Tourism Economics, 21, 957-976.

Ribeiro, L. C. S., \& Lopes, T. H. C. R. (2015). Características e similaridades do setor cultural nos munícipios e regiões metropolitanas brasileiras. Revista de Economia Contemporânea, 19(2), 307 330.

Ribeiro, L. C. S., Domingues, E. P., Perobelli, F. S., \& Hewings, G. J. D. (2018). Structuring investment and regional inequalities in the Brazilian Northeast. Regional Studies, 52(5), 727-739.

Ribeiro, L. C. S., Lopes, T. H. C. R., Montenegro, R. L., \& Andrade, J. R. L. (2017). Employment dynamics in the Brazilian tourism sector (2006-2015). Tourism Economics, 24(4), 418-433.

Rocha, F. (2007). Produtividade do trabalho e mudança estrutural nas indústrias brasileiras extrativa e de transformação, 1970-2001. Revista de Economia Política, 27(2), 221-241.

Sá, H. A., Morais, L., \& Campos, C. S. S. (2015). Que desenvolvimento é esse? Análise da expansão do agronegócio da soja na área do MATOPIBA a partir de uma perspectiva furtadiana. In Anais do XXI Congresso Brasileiro de Economia. Curitiba.

Santos, F., Ribeiro, L. C. S., \& Silveira, E. J. G. (2018). Characteristics of tourism activities in Brazilian municipalities in 2015. Brazilian Journal of Tourism Research, 12(2), 65-82.

Serigati, F., Rodrigues, R. M., Possamai, R., \& Vieira Filho, J. E. R. (2017). O mercado de trabalho na fronteira do agronegócio: quanto a dinâmica no Matopiba difere das regiões mais tradicionais? (Texto para Discussão, No. 2277). Rio de Janeiro: IPEA.

Silva, J. C. C. (2011). A análise de componentes de variação (Shift-Share). In J. S. Costa, T. P. Dentinho \& P. Nijkamp (Eds.), Compêndio de economia regional: métodos e técnicas de análise regional. Cascais, Portugal: Princípia.

Silveira Neto, R. D. M., \& Azzoni, C. R. (2011). Non-spatial government policies and regional income inequality in Brazil. Regional Studies, 45(4), 453-461.

Silveira-Neto, R. M., \& Azzoni, C. R. (2012). Social policy as regional policy: Market and nonmarket factors determining regional inequality. Journal of Regional Science, 52(3), 433-450.

Simões, R. F. (2006). Métodos de análise regional e urbana: diagnóstico aplicado ao planejamento. In C. C. Diniz \& M. A. Crocco (Eds.), Economia regional e urbana: contribuições teóricas recentes (1. ed.). Belo Horizonte: Editora da UFMG.

Souza, L. R. S., Ribeiro, L. C. S., \& Lopes, T. H. C. R. (2018). A dinâmica da indústria nos municípios de Sergipe (2010-2015). In Anais do XVI Encontro Nacional da Associação Brasileira de Estudos Regionais e Urbanos. São Paulo: ABER.

Williamson, J. G. (1965). Regional inequality and the process of national development: a description of the patterns. Economic Development and Cultural Change, 13(4), 1-84.

JEL: C38; R58; Q10 